\title{
ON JOINT HYPONORMALITY OF OPERATORS
}

\author{
AMEER ATHAVALE
}

(Communicated by John B. Conway)

\begin{abstract}
A notion of joint hyponormality is introduced for a collection of bounded linear operators on a separable Hilbert space.
\end{abstract}

It is the purpose of this note to introduce a notion of joint hyponormality for a collection of bounded linear operators on a separable Hilbert space $\mathscr{H}$ in a way that will meet the following conditions.

(a) The notion of joint hyponormality will be in some sense a natural generalization of the notion of hyponormality for a single operator.

(b) The notion will be at least as strong as requiring that the linear span of a given collection of operators consists of hyponormal operators.

(c) The notion will relate in a reasonable way to the questions pertaining to commuting normal extensions of commuting operators.

In some ways, the present note continues the investigations started in [3], but in addition it paraphrases the statements of some known results and open problems in subnormal operator theory in terms of joint hyponormality. We begin by fixing some notation. The set of bounded linear operators on a Hilbert space $\mathscr{H}$ will be denoted by $\mathscr{B}(\mathscr{H})$, while $\mathscr{H}^{(m)}$ will stand for the direct sum of $\mathscr{H}$ with itself $m$ times. An $m \times m$ operator matrix $\mathscr{A}=\left(A_{i j}\right)$ will be the matrix with the operator $A_{i j}$ as the (ij)th entry. The commutator $A B-B A$ of two elements $A$ and $B$ in $\mathscr{B}(\mathscr{H})$ will be denoted by $[A, B]$. Recall that an operator $H$ in $\mathscr{B}(\mathscr{H})$ is called hyponormal if the self-commutator $\left[H^{*}, H\right]$ of $H$ is positive. The set of all hyponormal operators in $\mathscr{B}(\mathscr{H})$ will be denoted by $H N(\mathscr{H})$, while $\operatorname{LS}\left\{A_{1}, \ldots, A_{m}\right\}$ will stand for the linear span of operators $A_{1}, \ldots, A_{m}$ in $\mathscr{B}(\mathscr{H})$. All the Hilbert spaces occurring below are separable.

DEFINITION 1. Let $H_{1}, \ldots, H_{m}$ be in $\mathscr{B}(\mathscr{H})$. We say that the tuple $\widetilde{H}=$ $\left(H_{1}, \ldots, H_{m}\right)$ is hyponormal, or that the operators $H_{1}, \ldots, H_{m}$ are jointly hyponormal, if $\mathscr{C}(\widetilde{H})=\left(\left[H_{j}^{*}, H_{i}\right]\right) \geq 0$ on $\mathscr{H}^{(m)}$.

There are several remarks to be made regarding Definition 1.

REMARK $0 . \mathscr{C}(\widetilde{H})=\left(\left[H_{j}^{*}, H_{i}\right]\right)$ is selfadjoint for any $m$ operators $H_{1}, \ldots, H_{m}$ in $\mathscr{B}(\mathscr{H})$.

REMARK 1. Let $\tilde{H}=\left(H_{1}, \ldots, H_{m}\right)$ be hyponormal and let $x_{1}, \ldots, x_{m}$ be in $\mathscr{H}$. Letting $\tilde{x}$ be the transpose $\left(x_{1}, \ldots, x_{m}\right)^{T}$ of the row vector $\left(x_{1}, \ldots, x_{m}\right)$, we have that $\left\langle\left(\left[H_{j}^{*}, H_{i}\right]\right) \tilde{x}, \tilde{x}\right\rangle_{\mathscr{P}(m)} \geq 0$. It is easily seen that this is the same as

$$
\sum_{i, j=1}^{m}\left\langle H_{i} x_{j}, H_{j} x_{i}\right\rangle_{\mathscr{H}}-\left\|\sum_{i=1}^{m} H_{i}^{*} x_{i}\right\|_{\mathscr{H}}^{2} \geq 0 .
$$

Received by the editors February 20, 1987.

1980 Mathematics Subject Classification (1985 Revision). Primary 47B20.

(C) 1988 American Mathematical Society $0002-9939 / 88 \$ 1.00+\$ .25$ per page 
REMARK 2. The following facts are obvious either from Definition 1 or relation (1).

(a) For $m=1$, Definition 1 is the usual definition of hyponormality for a single operator $H$ on $\mathscr{H}$.

(b) Hyponormality of $\widetilde{H}$ is not affected by permuting the operators $H_{i}$.

(c) If $\widetilde{H}$ is hyponormal, then so is any subtuple of $\widetilde{H}$.

(d) If $\widetilde{H}=\left(H_{1}, \ldots, H_{m}\right)$ is hyponormal, then so is $\left(k_{1} H_{1}, \ldots, k_{m} H_{m}\right)$ for $k_{1}, \ldots, k_{m}$ in $\mathbb{C}$, the complex field. (Replace $x_{j}$ by $\bar{k}_{j} x_{j}$ in (1).)

(e) If $\widetilde{H}=\left(H_{1}, \ldots, H_{m}\right)$ is hyponormal, then so is $\left(H_{1}-k_{1} I, \ldots, H_{m}-k_{m} I\right)$, where $k_{1}, \ldots, k_{m}$ are in $\mathbb{C}$ and $I$ is the identity operator on $\mathscr{H}$.

(f) If $\widetilde{H}=\left(H_{1}, \ldots, H_{m}\right)$ is hyponormal and $N$ is any normal operator commuting with each $H_{i}$, then $\left(N H_{1}, \ldots, N H_{m}\right)$ is hyponormal too. (Use the Fuglede Theorem [5].)

(g) If $\widetilde{H}=\left(H_{1}, \ldots, H_{m}\right)$ is hyponormal, then so is $\left(U^{*} H_{1} U, \ldots, U^{*} H_{m} U\right)$, where $U$ is any unitary operator in $\mathscr{B}(\mathscr{H})$.

(h) If $H \in H N(\mathscr{H})$, then $(H, \ldots, H)$ is hyponormal.

LEMMA 1. If $\mathscr{A}=\left(A_{i j}\right) \geq 0$ on $\mathscr{H}^{(m)}$, then $\mathscr{A}$ can be expressed as $\mathscr{A}=$ $\left(\sum_{k=1}^{m} A_{i}^{(k)} A_{j}^{(k)^{*}}\right)$ for some operators $A_{i}^{(k)}(1 \leq k, i \leq m)$ in $\mathscr{B}(\mathscr{H})$.

ProOF. This follows straight from Lemma 3.1, Chapter IV in [9].

LEMMA 2. If $\mathscr{A}=\left(A_{i j}\right) \geq 0$ on $\mathscr{H}^{(2)}$ and either $A_{11}=0$ or $A_{22}=0$, then $A_{12}=A_{21}=0$.

ProOF. This is obvious from Lemma 1.

REMARK 3. If $\widetilde{H}$ is hyponormal, then $\mathscr{C}(\widetilde{H}) \geq 0$ on $\mathscr{H}^{(m)}$ and this in turn implies that the diagonal entries of $\mathscr{C}(\widetilde{H})$ are positive operators. Hence if $\widetilde{H}=$ $\left(H_{1}, \ldots, H_{m}\right)$ is hyponormal, then each $H_{i}$ is in $H N(\mathscr{H})$.

REMARK 4. If $H, N$ in $\mathscr{B}(\mathscr{H})$ are such that $H$ is hyponormal, $N$ is normal and $H$ commutes with $N$, then $(H, N)$ is hyponormal. In fact,

$$
\mathscr{C}((H, N))=\left(\begin{array}{cc}
H^{*} H-H H^{*} & 0 \\
0 & 0
\end{array}\right) \geq 0 .
$$

(Note the use of the Fuglede Theorem [5].) Conversely, if $(H, N)$ is hyponormal and $N$ is normal, then $H$ commutes with $N$. In fact, $\mathscr{C}((H, N)) \geq 0$ implies that

$$
\left(\begin{array}{cc}
H^{*} H-H H^{*} & N^{*} H-H N^{*} \\
H^{*} N-N H^{*} & 0
\end{array}\right) \geq 0,
$$

which forces $N^{*} H=H N^{*}$ by Lemma 2, and this in turn implies $H N=N H$ by the Fuglede Theorem. With reference to Corollary 2.8 in [3], this observation yields the following: If $H$ is hyponormal and $N$ is normal, then $(H, N)$ is hyponormal if and only if $\operatorname{LS}\{H, N\} \subset H N(\mathscr{H})$.

REMARK 5. If $H_{1}, \ldots, H_{m}$ are in $\mathscr{B}(\mathscr{H})$ and Lat $H_{i}$ denotes the lattice of invariant subspaces of $H_{i}$, then hyponormality of $\widetilde{H}$ implies that $\left(H_{1} / \mathscr{L}, \ldots, H_{m} / \mathscr{L}\right)$ is hyponormal for any $\mathscr{L} \in \bigcap_{i=1}^{m}$ Lat $H_{i}$. This follows directly from relation (1). 
PROPOSITION 1. If $H_{1}, \ldots, H_{m}$ are in $\mathscr{B}(\mathscr{H})$ and $\widetilde{H}=\left(H_{1}, \ldots, H_{m}\right)$ is hyponormal, then $\operatorname{LS}\left\{H_{1}, \ldots, H_{m}\right\} \subset H N(\mathscr{H})$.

PROOF. For $k_{i} \in \mathbb{C}$,

$$
\left[\left(\sum_{i=1}^{m} k_{i} H_{i}\right)^{*},\left(\sum_{i=1}^{m} k_{i} H_{i}\right)\right]=\sum_{i, j=1}^{m} k_{i} \bar{k}_{j}\left[H_{j}^{*}, H_{i}\right] .
$$

Now if $\mathscr{C}(\tilde{H}) \geq 0$, then, for any $x_{1}, \ldots, x_{m}$ in $\mathscr{H}$,

$$
\sum_{i, j=1}^{m}\left\langle\left[H_{j}^{*}, H_{i}\right] x_{j}, x_{i}\right\rangle_{\mathscr{H}} \geq 0 .
$$

Letting $x_{j}=\bar{k}_{j} x$ for a fixed vector $x$ in $\mathscr{H}$, we see that $\mathscr{C}(\tilde{H}) \geq 0$ implies that

$$
\left\langle\left[\left(\sum_{i=1}^{m} k_{i} H_{i}\right)^{*},\left(\sum_{i=1}^{m} k_{i} H_{i}\right)\right] x, x\right\rangle_{\mathscr{\ell}} \geq 0 ;
$$

that is, $\operatorname{LS}\left\{H_{1}, \ldots, H_{m}\right\} \subset H N(\mathscr{H})$.

REMARK 6. At this stage, the condition of joint hyponormality looks too strong for the conclusion of Proposition 1. The above proof brings out the fact that the condition that $\operatorname{LS}\left\{H_{1}, \ldots, H_{m}\right\} \subset H N(\mathscr{H})$ is equivalent to $\langle\mathscr{C}(\tilde{H}) \tilde{x}, \tilde{x}\rangle_{\mathscr{H}(m)} \geq 0$ for every $\tilde{x}$ of the form $\left(\alpha_{1} x, \ldots, \alpha_{m} x\right)^{T}$, where $\alpha_{i} \in \mathbb{C}$ and $x \in \mathscr{H}$. However, the author does not know of an example of even two operators $H_{1}$ and $H_{2}$ in $\mathscr{B}(\mathscr{H})$ for which $\operatorname{LS}\left\{H_{1}, H_{2}\right\} \subset H N(\mathscr{H})$, but $\left(H_{1}, H_{2}\right)$ is not hyponormal. It is remarked that for $m$ operators $H_{1}, \ldots, H_{m}$ in $\mathscr{B}(\mathscr{H})$, the condition that $\operatorname{LS}\left\{H_{1}, \ldots, H_{m}\right\} \subset H N(\mathscr{H})$ is equivalent to requiring the principal minors of the scalar matrix $\left(\left\langle\left[H_{j}^{*}, H_{i}\right] x, x\right\rangle_{\mathscr{H}}\right)$ to be nonnegative for every $x$ in $\mathscr{H}$. (This observation is really a generalization of Proposition 2.5 in [3].)

REMARK 7. If for $H_{1}, \ldots, H_{m}$ in $\mathscr{B}(\mathscr{H}), H_{i}^{*} H_{j}=H_{j} H_{i}^{*}$ for $i \neq j$, and each $H_{i}$ is hyponormal, then $\mathscr{C}(\widetilde{H})$ is a diagonal matrix with positive operator entries along the diagonal, and hence is positive. By Proposition 1, this yields Proposition 2.3 in [3]. The consideration of $H_{1}=H_{2}=$ unilateral shift of multiplicity one shows that the condition of hyponormality of $\left(H_{1}, H_{2}\right)$ covers a wider class of hyponormal operators than the condition $H_{1}^{*} H_{2}=H_{2} H_{1}^{*}$ to imply that $\mathrm{LS}\left\{H_{1}, H_{2}\right\} \subset H N(\mathscr{H})$. (For the example at hand, using an orthonormal basis $\left\{e_{0}, e_{1}, \ldots\right\}$ of $\mathscr{H}, \mathscr{C}\left(\left(H_{1}, H_{2}\right)\right)$ can be represented as $\mathscr{C}\left(\left(H_{1}, H_{2}\right)\right)=\left(A_{i j}\right)$, where $A_{11}=A_{12}=A_{21}=A_{22}=$ the infinite matrix with entry 1 in the (11)th place (i.e. in the first column and the first row) and zeros elsewhere. It is clear that $\mathscr{C}\left(\left(H_{1}, H_{2}\right)\right) \geq 0$. See also part (h) of Remark 2.)

REMARK 8 . We did not require the operators $H_{1}, H_{2}$ to be commuting in the definition of hyponormality of $\left(H_{1}, H_{2}\right)$. Example 2.4 in [3] shows that we can have two noncommuting hyponormals $H_{1}, H_{2}$ such that $\left(H_{1}, H_{2}\right)$ is hyponormal.

REMARK 9. If $W$ is a hyponormal unilateral weighted shift, then it is easy to verify that every power of $W$ is hyponormal. Peng Fan [4] gave an example of a $W$ for which $\operatorname{LS}\left\{W, W^{2}\right\} \not \subset H N(\mathscr{H})$. In view of Proposition 1, this shows that we can have two commuting hyponormals whose tuple is not hyponormal. 
We now come to the questions involving subnormal operators. Recall that an operator $S$ on $\mathscr{H}$ is subnormal if it is the restriction of a normal operator $N$ to $\mathscr{H}$, $\mathscr{H}$ being invariant for $N$.

DEFINITION 2. If $S_{1}, \ldots, S_{m}$ are commuting operators in $\mathscr{B}(\mathscr{H})$, then $\widetilde{S}=$ $\left(S_{1}, \ldots, S_{m}\right)$ is said to have a commuting normal extension (or is said to be subnormal) if there exist commuting normal operators $N_{1}, \ldots, N_{m}$ on some Hilbert space $\mathscr{K} \supset \mathscr{H}$ such that $N_{i} \mathscr{H} \subset \mathscr{H}$ and $N_{i} / \mathscr{H}=S_{i}$ for all $i$.

PROPOSITION 2. Let $S_{1}, \ldots, S_{m}$ be commuting operators in $\mathscr{B}(\mathscr{H})$. If $\widetilde{S}=$ $\left(S_{1}, \ldots, S_{m}\right)$ has a commuting normal extension, then $\widetilde{S}$ is hyponormal.

PROOF. If $N_{j}$ is the normal extension of $S_{j}$, write

$$
N_{j}=\left(\begin{array}{cc}
S_{j} & X_{j} \\
0 & Y_{j}
\end{array}\right)
$$

Here $X_{j}: \mathscr{K} \ominus \mathscr{H} \rightarrow \mathscr{H}$ and $Y_{j}: \mathscr{K} \ominus \mathscr{H} \rightarrow \mathscr{K} \ominus \mathscr{H}$ are some operators and $\mathscr{K} \ominus \mathscr{H}$ denotes the orthogonal complement of $\mathscr{H}$ in $\mathscr{K}$. Equating $N_{j}^{*} N_{i}$ and $N_{i} N_{j}^{*}$ (which is justified by the Fuglede Theorem), we get $\left[S_{j}^{*}, S_{i}\right]=X_{i} X_{j}^{*}$ so that $\mathscr{C}(S)=\left(\left[S_{j}^{*}, S_{i}\right]\right)=\left(X_{i} X_{j}^{*}\right) \geq 0$ on $\mathscr{H}^{(m)}$.

PROPOSITION 3. Let $S$ be in $\mathscr{B}(\mathscr{H})$. Then $S$ is subnormal if and only if $\left(I, S, \ldots, S^{p-1}\right)$ is hyponormal for every $p \geq 1$.

PROOF. The operator $S$ is subnormal if and only if the Halmos-Bram conditions [2] hold; that is, if and only if

$$
\sum_{j, k=0}^{p-1}\left\langle S^{j} x_{k}, S^{k} x_{j}\right\rangle_{\mathscr{C}} \geq 0
$$

for all $p \geq 1$ and any $x_{j}$ in $\mathscr{H}$. Write

$$
\begin{aligned}
& \sum_{j, k=0}^{p-1}\left\langle S^{j} x_{k}, S^{k} x_{j}\right\rangle_{\mathscr{H}}=\sum_{j, k=0}^{p-1}\left\langle\left(S^{* k} S^{j}-S^{j} S^{* k}\right) x_{k}, x_{j}\right\rangle_{\mathscr{H}} \\
& \quad+\sum_{j, k=0}^{p-1}\left\langle S^{j} S^{* k} x_{k}, x_{j}\right\rangle_{\mathscr{H}}=\left\langle\mathscr{C}\left(\left(I, S, \ldots, S^{p-1}\right)\right) \tilde{x}, \tilde{x}\right\rangle_{\mathscr{L}(p)} \\
& +\left\|\sum_{k=0}^{p-1} S^{* k} x_{k}\right\|_{\mathscr{H}}^{2}, \quad \text { where } \tilde{x}=\left(x_{0}, \ldots, x_{p-1}\right)^{T} .
\end{aligned}
$$

Thus if $\left(I, S, \ldots, S^{p-1}\right)$ is hyponormal for every $p \geq 1$, then the Halmos-Bram conditions hold. Conversely, if $S$ is subnormal, then $\left(I, S, \ldots, S^{p-1}\right)$ has a commuting normal extension for every $p \geq 1$ and this implies, by Proposition 2, that $\left(I, S, \ldots, S^{p-1}\right)$ is hyponormal for every $p \geq 1$.

REMARK 10. The Halmos-Bram conditions for a single operator $S$ were generalized by Ito [6] to the case of $m$ commuting operators $S_{1}, \ldots, S_{m}$. Ito's result combined with parts (b) and (c) of Remark 2 yields that the tuple $S=\left(S_{1}, \ldots, S_{m}\right)$ of $m$ commuting operators on $\mathscr{H}$ is subnormal if and only if all possible tuples whose components are products of nonnegative powers of $S_{i}$ are hyponormal. 
Let $\left\{S_{\gamma}\right\}_{\gamma \in \Gamma}$ be a family of commuting operators in $\mathscr{B}(\mathscr{H})$. We say that $\left\{S_{\gamma}\right\}_{\gamma \in \Gamma}$ has a commuting normal extension if $\left(S_{\gamma_{1}}, \ldots, S_{\gamma_{m}}\right)$ has a commuting normal extension for every finite index set $\left\{\gamma_{1}, \ldots, \gamma_{m}\right\} \subset \Gamma$. If $\left\{S_{\gamma}\right\}_{\gamma \in \Gamma}$ is any family of operators in $\mathscr{B}(\mathscr{H})$, then $\left\{S_{\gamma}\right\}_{\gamma \in \Gamma}$ is said to be jointly quasinormal if $\left\{S_{\gamma}, S_{\gamma}^{*} S_{\gamma}\right\}_{\gamma \in \Gamma}$ is a commutative family. In view of parts (b) and (c) of Remark 2, it then seems sensible to make the following definition in the same vein.

DEFINITION 3. Let $\left\{S_{\gamma}\right\}_{\gamma \in \Gamma}$ be a family of operators in $\mathscr{B}(\mathscr{H})$. Then $\left\{S_{\gamma}\right\}_{\gamma \in \Gamma}$ is said to be jointly hyponormal if $\left(S_{\gamma_{1}}, \ldots, S_{\gamma_{m}}\right)$ is hyponormal for every finite index set $\left\{\gamma_{1}, \ldots, \gamma_{m}\right\} \subset \Gamma$.

REMARK 11. Making crucial use of a result of Yoshino [12], A. Lubin [7] showed that if $\left\{S_{\gamma}\right\}_{\gamma \in \Gamma}$ is a family of jointly quasinormal operators, then $\left\{S_{\gamma}\right\}_{\gamma \in \Gamma}$ has a commuting normal extension. Using Lemma 2, it is easy to see that this can be described as follows: Let $\left\{S_{\gamma}\right\}_{\gamma \in \Gamma}$ be a commutative family of operators in $\mathscr{B}(\mathscr{H})$. Then $\left\{S_{\gamma}\right\}_{\gamma \in \Gamma}$ has a commuting normal extension if $\left(S_{\alpha}, S_{\beta}^{*} S_{\beta}\right)$ is hyponormal for all $\alpha, \beta$ in $\Gamma$.

We next come to the discussion of some open questions in subnormal operator theory and their relation to the concept of joint hyponormality.

Question 1. Is every polynomially hyponormal operator subnormal? That is, if $H \in \mathscr{B}(\mathscr{H})$ is such that $p(H)$ is hyponormal for every polynomial $p$, is it true that $H$ is subnormal?

Note that examples of power-hyponormal nonsubnormal operators are already known $[\mathbf{4}, \mathbf{8}]$. In view of Proposition 2, Question 1 can be reformulated as follows: If $\operatorname{LS}\left\{I, H, \ldots, H^{p-1}\right\} \subset H N(\mathscr{H})$ for every $p \geq 1$, is it true that $\left(I, H, \ldots, H^{k-1}\right)$ is hyponormal for every $k \geq 1$ ? At this stage the reader is referred back to Remark 6.

Question 2. This is the analogue of Question 1 for more than one operator. In particular, if $H_{1}$ and $H_{2}$ are two commuting operators in $\mathscr{B}(\mathscr{H})$ such that $p\left(H_{1}, H_{2}\right)$ is hyponormal for every two-variable polynomial $p$, is it true that $\left(H_{1}, H_{2}\right)$ has a commuting normal extension?

For the corresponding formulation of this question in terms of joint hyponormality we refer the reader to Remark 10 .

Question 3. A. Lubin [7] asks whether for two commuting operators $S_{1}, S_{2}$ in $\mathscr{B}(\mathscr{H})$, subnormality of $p\left(S_{1}, S_{2}\right)$ for every two-variable polynomial $p$ implies that $\left(S_{1}, S_{2}\right)$ has a commuting normal extension. It is obvious that an affirmative answer to Question 2 would yield an affirmative answer to Question 3.

In the following, we make a few observations involving multicyclic hyponormals.

DEFINITION 4. An operator $H$ in $\mathscr{B}(\mathscr{H})$ is said to be $m$-multicyclic if there exists a finite number of vectors $f_{1}, \ldots, f_{k}$ in $\mathscr{H}$ such that the linear span of the family $\left\{r(H) f_{j}: r\right.$ is a rational function with poles off $\left.\sigma(H) ; 1 \leq j \leq k\right\}$ is dense in $\mathscr{H}$ and $m$ is the least such integer $k$.

It is known by the Berger-Shaw Theorem [1] that any $m$-multicyclic operator has a trace-class self-commutator. Also, it is easy to see that any cyclic operator is 1-multicyclic.

PROPOSITION 4. Let $H_{1}$ and $H_{2}$ be two operators in $\mathscr{B}(\mathscr{H})$ such that $H_{1}$ is $m_{1}$-multicyclic and $H_{2}$ is $m_{2}$-multicyclic. If $\operatorname{LS}\left\{H_{1}, H_{2}\right\} \subset H N(\mathscr{H})$, then $\left[H_{j}^{*}, H_{i}\right]$ 
is trace-class and

$\left|\operatorname{Trace}\left[H_{j}^{*}, H_{i}\right]\right| \leq(1 / \pi)\left(m_{i} m_{j} \operatorname{Area}\left(\sigma\left(H_{i}\right)\right) \operatorname{Area}\left(\sigma\left(H_{j}\right)\right)\right)^{1 / 2} ; \quad 1 \leq i, j \leq 2$. If $\left(H_{1}, H_{2}\right)$ is hyponormal, then we have as well

$$
\left\|\left[H_{j}^{*}, H_{i}\right]\right\|_{2} \leq(1 / \pi)\left(m_{i} m_{j} \operatorname{Area}\left(\sigma\left(H_{i}\right)\right) \operatorname{Area}\left(\sigma\left(H_{j}\right)\right)\right)^{1 / 2}
$$

for $1 \leq i, j \leq 2$; where $\|\cdot\|_{2}$ denotes the Hilbert-Schmidt norm.

ProOF. Suppose LS $\left\{H_{1}, H_{2}\right\} \subset H N(\mathscr{H})$. If $\left(e_{j}\right)_{j}$ is an orthonormal basis of $\mathscr{H}$, then by Remark 6 , we have that

$$
\left|\left\langle\left[H_{2}^{*}, H_{1}\right] e_{n}, e_{n}\right\rangle\right|^{2} \leq\left\langle\left[H_{1}^{*}, H_{1}\right] e_{n}, e_{n}\right\rangle\left\langle\left[H_{2}^{*}, H_{2}\right] e_{n}, e_{n}\right\rangle
$$

for any fixed $n$. Taking square roots, summing over $n$ and applying Hölder's inequality, we obtain

$$
\left|\operatorname{Trace}\left[H_{2}^{*}, H_{1}\right]\right| \leq\left(\operatorname{Trace}\left[H_{1}^{*}, H_{1}\right]\right)^{1 / 2}\left(\operatorname{Trace}\left[H_{2}^{*}, H_{2}\right]\right)^{1 / 2} .
$$

If $\left(H_{1}, H_{2}\right)$ is hyponormal, then fix $m$ and $n$. The nonnegativity of

$$
\left\langle\mathscr{C}\left(\left(H_{1}, H_{2}\right)\right) \tilde{x}, \tilde{x}\right\rangle_{\mathscr{H}(2)}
$$

for any $\tilde{x}=\left(\alpha_{1} e_{n}, \alpha_{2} e_{m}\right)^{T}$, with $\alpha_{1}$ and $\alpha_{2}$ in $\mathbb{C}$, is equivalent to

$$
\left|\left\langle\left[H_{2}^{*}, H_{1}\right] e_{n}, e_{m}\right\rangle\right|^{2} \leq\left\langle\left[H_{1}^{*}, H_{1}\right] e_{n}, e_{n}\right\rangle\left\langle\left[H_{2}^{*}, H_{2}\right] e_{m}, e_{m}\right\rangle \text {. }
$$

Summing over $n$ and $m$ and taking square roots, we have that

$$
\left\|\left[H_{2}^{*}, H_{1}\right]\right\|_{2} \leq\left(\operatorname{Trace}\left[H_{1}^{*}, H_{1}\right]\right)^{1 / 2}\left(\operatorname{Trace}\left[H_{2}^{*}, H_{2}\right]\right)^{1 / 2}
$$

as well. The desired conclusion for $i \neq j$ then follows by using the well-known estimates given by the Berger-Shaw Theorem. The cases $i=j$ are obvious.

COROLLARY 1. Let $S_{1}$ and $S_{2}$ be two commuting subnormals in $\mathscr{B}(\mathscr{H})$ such that $S_{1}$ is cyclic and $S_{2}$ is $m$-multicyclic. Then $\left[S_{2}^{*}, S_{1}\right]$ is trace-class and both | Trace $\left[S_{2}^{*}, S_{1}\right] \mid$ and $\left\|\left[S_{2}^{*}, S_{1}\right]\right\|_{2}$ are less than or equal to

$$
(1 / \pi)\left(m \operatorname{Area}\left(\sigma\left(S_{1}\right)\right) \operatorname{Area}\left(\sigma\left(S_{2}\right)\right)\right)^{1 / 2} .
$$

PROOF. Since $S_{1}$ is cyclic, $\left(S_{1}, S_{2}\right)$ has a commuting normal extension by a result of Yoshino [11]. By Proposition 2 and Proposition 1, it follows that $\left(S_{1}, S_{2}\right)$ is hyponormal and $\operatorname{LS}\left\{S_{1}, S_{2}\right\} \subset H N(\mathscr{H})$. The rest is obvious.

It is finally pointed out that the concept of a hyponormal tuple $(\tilde{X}, Y)=$ $\left(X_{1}, \ldots, X_{m}, Y\right)$ was introduced by $\mathrm{Xia}$ in [10], where $X_{1}, \ldots, X_{m}$ are commuting selfadjoint operators in $\mathscr{B}(\mathscr{H})$ and $Y \in \mathscr{B}(\mathscr{H})$. We merely comment that our definition of joint hyponormality is applicable to any collection of operators in $\mathscr{B}(\mathscr{H})$ and for $m$ commuting selfadjoint operators $X_{1}, \ldots, X_{m}$ in $\mathscr{B}(\mathscr{H})$, hyponormality of $(\tilde{X}, Y)$ in our sense implies hyponormality in the sense of Xia in an obvious way.

ACKNOWLEDGMENTS. The author would like to thank John B. Conway for his constant guidance throughout the preparation of this note. Thanks are also due to Vern Paulsen with whom the author had some fruitful discussions.

This work was supported by NSF grant DMS-83-20426. 


\section{BIBLIOGRAPHY}

1. C. A. Berger and B. I. Shaw, Self-commutators of multicyclic hyponormals are always trace class, Bull. Amer. Math. Soc. 79 (1973), 1193-1199.

2. J. Bram, Subnormal operators, Duke Math. J. 22 (1955), 75-94.

3. J. B. Conway and W. Szymanski, Linear combinations of hyponormal operators, Rocky Mountain J. Math. (to appear).

4. P. Fan, A note on hyponormal weighted shifts, Proc. Amer. Math. Soc. 92 (1984), 271-272.

5. B. Fuglede, A commutativity theorem for normal operators, Proc. Mat. Acad. Sci. 36 (1950), 35-40.

6. T. Ito, On the commutative family of subnormal operators, J. Fac. Sci. Hokkaido Univ. 14 (1958), 1-5.

7. A. Lubin, Weighted shifts and commuting normal extension, J. Austral. Math. Soc. 27 (1979), 17-26.

8. J. G. Stampfli, Hyponormal operators and spectral density, Trans. Amer. Math. Soc. 117 (1965), 469-476.

9. M. Takesaki, Theory of operator algebras. I, Springer-Verlag, New York, 1979.

10. D. Xia, On the semi-hyponormal n-tuple of operators, Integral Equations Operator Theory 6 (1983), 879-898.

11. T. Yoshino, Subnormal operators with a cyclic vector, Tôhoku Math. J. 21 (1969), 47-55.

12. - , On commuting extensions of nearly normal operators, Tôhoku Math. J. 25 (1973), 263272.

DEPARTMENT OF MATHEMATICAL SCIENCES, INDIANA UNIVERSITY-PURDUE UNIVERSITY, P. O. BOX 647, INDIANAPOLIS, INDIANA 46223 